\title{
Continuous Wavelet Transform Based Frequency Dispersion Compensation Method for Electromagnetic Time-Reversal Imaging
}

DOI:

10.1109/TAP.2016.2647594

\section{Document Version}

Accepted author manuscript

Link to publication record in Manchester Research Explorer

Citation for published version (APA):

Abduljabbar, A., Yavuz, M. E., Costen, F., Himeno, R., \& Yokota, H. (2017). Continuous Wavelet Transform Based Frequency Dispersion Compensation Method for Electromagnetic Time-Reversal Imaging. IEEE Transactions on Antennas and Propagation, 65(3), 1321-1329. https://doi.org/10.1109/TAP.2016.2647594

\section{Published in:}

IEEE Transactions on Antennas and Propagation

\section{Citing this paper}

Please note that where the full-text provided on Manchester Research Explorer is the Author Accepted Manuscript or Proof version this may differ from the final Published version. If citing, it is advised that you check and use the publisher's definitive version.

\section{General rights}

Copyright and moral rights for the publications made accessible in the Research Explorer are retained by the authors and/or other copyright owners and it is a condition of accessing publications that users recognise and abide by the legal requirements associated with these rights.

\section{Takedown policy}

If you believe that this document breaches copyright please refer to the University of Manchester's Takedown Procedures [http://man.ac.uk/04Y6Bo] or contact uml.scholarlycommunications@manchester.ac.uk providing relevant details, so we can investigate your claim.

\section{OPEN ACCESS}




\title{
Continuous Wavelet Transform Based Frequency Dispersion Compensation Method for Electromagnetic Time-Reversal Imaging
}

\author{
Ammar M. Abduljabbar, Student Member, IEEE, Mehmet E. Yavuz, Fumie Costen, Senior Member, IEEE, \\ Ryutaro Himeno, Hideo Yokota
}

\begin{abstract}
The invariance of wave equations in lossless media allows the Time Reversal (TR) technique to spatio-temporally refocus back-propagated signals in a given ultrawideband imaging scenario. However, the existence of dispersion and loss in the propagation medium breaks this invariance and the resultant TR focusing exhibits frequency and propagation duration dependent degradation. We propose an algorithm based on the continuous wavelet transform that tackles this degradation to improve focusing resolution under such conditions. The developed algorithm has been successfully applied to the scenario for localization of lung cancer.
\end{abstract}

Index Terms-Time reversal (TR), continuous wavelet transform (CWT), dispersive media, attenuation compensation.

\section{INTRODUCTION}

$\mathbf{L}$ OCALIZATION of targets behind structures or within obstructed media is of great interest in many microwave imaging applications such as detection of landmines [1], explosives [2], subsurface imaging [3] or tumors in the body [4] (e.g. breast [5], brain [6] and lung [7].)

A recent method in the microwave imaging which has been introduced to detect and localize objects is the Time Reversal (TR) technique [8]. In a lossless medium, TR states that for every wave component propagating away from a source point following a certain path, there is a corresponding time-reversed wave that can trace the same path back to the original point of the source due to invariance of Maxwell's equations to time. Conventional TR technique uses a set of antennas, called TR Array (TRA), to receive, record and timereverse the reflected signal from the object(s). Time-reversed signals at each TRA antenna are sent back to the medium to focus around the original object location. One important property of TR is that it achieves super-resolution by utilizing the multipath propagation in the medium as discussed in [9], [10], [11]. On the other hand, as with other microwave imaging techniques, TR suffers from dispersion and losses in the propagating medium [12], [13] where TR invariance

A. M. Abduljabbar, and F. Costen are with the School of Electrical and Electronic Engineering, The University of Manchester, U.K. (email: fumie.costen@manchester.ac.uk)

M. E. Yavuz, Hillsboro, Oregon, USA. (email: yavuz.5@osu.edu)

R. Himeno is Advanced Center for Computing and Communication, RIKEN, Saitama, Japan

H. Yokota and F. Costen are with the Image Processing Research Team, Center for Advanced Photonics, RIKEN, Saitama, Japan.

Color versions of the figures in this paper are available online at http://ieeexplore.ieee.org. Additional research data supporting this publication are available from http://dx.doi.org/ repository at 10.15127/1.306001. of the wave equations is broken. While the additional phase shift undergone due to dispersion can be compensated by the TR process itself [12], attenuation which affects the signals in both the forward and backward propagation stages are not compensated. This attenuation ultimately degrades the focusing resolution of the localization. The earliest works on compensation of such attenuation involved a uniform absorption model over the entire bandwidth for acoustic propagation in skull [14], multipaths in ocean communication [15] and at a single frequency with the so-called decomposition of the TR operator method [16]. A more recent compensation technique using the Short-Time Fourier Transform (STFT) method takes frequency dependency into account and tries to improve the resolution focusing of the TR technique in dispersive and lossy media [12]. Although this method proved to be a good candidate for compensation, the inverse filters utilized in the method also amplify unwanted oscillations and perturbations in the received signals, which affect the performance of TR technique. STFT-based method of [12] produced the inverse filters by comparing the solution of the wave equation in the applied dispersive medium against a corresponding non-dispersive test medium by transmitting a pulse and observing it at various propagation distances for both media. The process of creating the inverse filters is empiric and time consuming. Later, the method in [12] is extended by adding a threshold approach to overcome the amplification of the noise in the inverse filter in [17]. Different window types and lengths are applied in [17] to obtain the optimum settings for the STFT technique. However, the work only applied in dispersive and homogeneous media which do not accurately represent the real-life applications. The inverse filters in [12] and [17] need the non-dispersive version of the propagation media to create the inverse filter which might be unknown in real-life scenarios. The STFT method uses a fixed time window length which provides a lower resolution in determining attenuation when compared with other size-adjustable window methods [18], [19]. In this paper, we propose a compensation method that uses adaptive-window scheme based on the Continuous Wavelet Transform (CWT). Our compensation method uses an inverse filter in the wavelet domain to overcome the attenuation and dispersion in the electromagnetic (EM) wave propagation due to the dispersive medium. Our method uses adjustable-length windows by applying long time-windows at low frequencies and short time-windows at high frequencies [20]. Therefore 
we do not need optimization of the time-window length as was carried out in [17]. The proposed inverse filters need only the complex permittivity of one dominant medium at the center frequency to create the inverse filter. creation of inverse filters in the STFT method requires more computation compared to those in the CWT, hence another advantage of CWT over STFT. To the best knowledge of the authors, such an adaptive compensation algorithm has not been considered elsewhere before. Therefore introduction of such an algorithm is considered as the main contribution of this paper. Numerical simulations have been chosen to validate the algorithm. The outline of the paper is as follows: First, the details of the proposed compensation method is described in Section II. Section III and Section IV provide the radio environment settings and the analysis of the numerical simulations for the canonical test and practical application respectively. Section $\mathrm{V}$ presents the conclusions.

\section{COMPENSATION OF DISPERSIVE ATTENUATION IN HUMAN TISSUES}

A dispersive and lossy medium acts as a low-pass filter for signals propagating through it. Thus, for compensation of such effects, inverse filters are needed. The real part of the medium dielectric permittivity $\epsilon$ causes a phase shift to the traveling waves during the forward propagation of the TR process. Thanks to the TR concept, this phase shift is inherently corrected in the backward propagation due to the fact that the signals in the backward propagation are phase-conjugated coherently along all bandwidth [21]. On the other hand, the imaginary part of $\epsilon$ causes inevitable signal attenuation. The attenuation is a function of time (in other words, duration the signal has traveled in the dispersive medium) and frequency. Note that the more the signal propagates in a dispersive medium the more it is attenuated (duration dependency). Similarly, for certain wave packet that has traveled in the dispersive medium, different frequency components undergo different attenuations (frequency dependency). Therefore the proposed method uses CWT technique which inherently incorporates both time and frequency variations as CWT suits well for this kind of problem. The wavelet function that we used for the CWT method is Morlet wavelet [22]. Morlet wavelet is considered in [19] as the proper choice for analyzing the propagated wave in attenuating and dispersive media. The mother wavelet of Morlet in time domain [23] is

$$
\Psi_{0}[n]=\frac{1}{\sqrt[4]{\pi}} e^{\jmath f_{\Psi} n \Delta t} e^{-\frac{(n \Delta t)^{2}}{2}}
$$

where $n$ is the time index, $\Delta t$ is the time step in seconds and $f_{\Psi}$ is the central frequency of the mother wavelet. Note that without loss of generality, we have chosen to work with discrete signals instead of the continuous ones, hence the notation of $\Psi_{0}[n]$ instead of $\Psi_{0}(t) . \Psi_{0}[n]$ is expressed in scaled-frequency domain as

$$
\Psi_{0}\left(a_{j} \omega_{k}\right)=\frac{1}{\sqrt[4]{\pi}} e^{-\frac{\left(a_{j} \omega_{k}-f_{\Psi}\right)^{2}}{2}}
$$

where $a_{j}$ is the dimensionless scaling factor of $a_{j}=a_{0} 2^{j \Delta_{j}}$ for $j=0,1, \ldots, J-1, a_{0}$ is the smallest scale, $\Delta_{j}$ is the scale step, $J$ is the largest scale $J=\left\lceil\frac{1}{\Delta_{j}} \log _{2}\left(\frac{N \Delta t}{a_{0}}\right)\right\rceil,\lceil\rceil$ represents the ceiling function, $N$ is the sampling points in the time domain signal, $k$ is frequency index and the angular frequency $\omega_{k}$ is [23]

$$
\omega_{k}=\left\{\begin{array}{cc}
0 & k=1 \\
\frac{2 \pi k}{N \Delta t} & 1<k \leq \frac{N}{2}+1 \\
-\frac{2 \pi k}{N \Delta t} & \frac{N}{2}+1<k \leq N
\end{array}\right.
$$

for $k=1,2, \ldots, N$. $a_{0}$ is dimensionless and it is set to the value of $\Delta t$ and $\Delta_{j}$ is set to 0.025 based on the value chosen in [23]. Both $\Psi_{0}[n]$ in (1) and $\Psi_{0}\left(a_{j} \omega_{k}\right)$ in (2) are complex. In order for the analyzing function $\Psi_{0}[n]$ to be called wavelet it needs to be admissible [24]. Admissibility is one of the necessary conditions of the wavelet transform and it is achieved when [24]

$$
\lim _{N \rightarrow \infty} \sum_{n=-N}^{N} \Psi_{0}[n]=0
$$

and

$$
\Psi_{0}\left(a_{j} \omega_{k}\right)=\left\{\begin{array}{cc}
\frac{1}{\sqrt[4]{\pi}} e^{-\frac{\left(a_{j} \omega_{k}-f_{\Psi}\right)^{2}}{2}} & \omega_{k}>0 \\
0 & \omega_{k} \leq 0 .
\end{array}\right.
$$

We added white Gaussian noise to each observed signal $x[n]$ at each TRA antenna. The average power of the additive noise is $20 \%$ of that of $x[n]$ which is chosen to mimic the system noise based on [25]. Our compensation method starts with conversion of the observed signal $x[n]$ in time domain to the wavelet domain. The CWT of $x[n]$ is the convolution of the wave function $\Psi^{*}\left(\frac{n}{a_{j}}\right)$ and $x[n]$ which is equivalent to the inverse Fourier transform of the product of $\Psi^{*}\left(a_{j} \omega_{k}\right)$ and $x[k]$ in the frequency domain where ${ }^{*}$ represents the complex conjugate, $\Psi\left(\frac{n}{a_{j}}\right)$ is

$$
\Psi\left(\frac{n}{a_{j}}\right)=\sqrt{\frac{\Delta t}{a_{j}}} \Psi_{0}\left(\frac{n}{a_{j}}\right)
$$

and $\Psi\left(a_{j} \omega_{k}\right)$ is

$$
\Psi\left(a_{j} \omega_{k}\right)=\sqrt{\frac{2 \pi a_{j}}{\Delta t}} \Psi_{0}\left(a_{j} \omega_{k}\right) .
$$

$\Psi\left(a_{j} \omega_{k}\right)$ represents the normalized $\Psi_{0}\left(a_{j} \omega_{k}\right)$ to guarantee that the CWTs at each $a_{j}$ are comparable to each other and to the CWTs of other time series [23]. $\Psi\left(a_{j} \omega_{k}\right)$ satisfies

$$
\sum_{k=1}^{N}\left|\Psi\left(a_{j} \omega_{k}\right)\right|^{2}=N \text {. }
$$

The CWT for the observed signal $x[n]$ at each TRA antenna is

$$
X\left[n, a_{j}\right]=\sum_{k=1}^{N}\left(\sum_{i=1}^{N} x[i] e^{-\frac{2 \pi}{N} i k} \Psi^{*}\left(a_{j} \omega_{k}\right)\right) e^{\jmath \frac{2 \pi}{N} n k} .
$$

The CWT method applies a long window (large $a_{j}$ ) at the lower end of the spectrum (low frequency) and a short window (small $a_{j}$ ) at the higher end of the spectrum (high frequency) to provide a perfect filter for time-dependent implementations in terms of time and frequency localization. Furthermore 
the CWT method provides us with an improved separation between the unwanted noise and our signal [19]. Next step is applying our inverse filter to $X\left[n, a_{j}\right]$ in the wavelet domain to compensate the attenuation caused by the dispersive medium. In order to derive the inverse filter, we need to calculate the attenuation. The solution of Maxwell equations for a plane wave propagating in a dispersive medium [26] is

$$
E[n]=e^{\jmath 2 \pi f \cdot n \Delta t} e^{-\jmath 2 \pi f \sqrt{\epsilon \mu} d[n]}
$$

where $f$ is the frequency of interest, $d[n]$ is the distance between the excitation and the observation, $\mu=\mu_{0} \mu_{r}$ is the permeability of the medium, $\mu_{0}$ is the free space permeability and $\mu_{r}$ is the relative permeability which is set to 1 as a nonmagnetically dispersive medium is assumed, $\epsilon=\epsilon_{0} \epsilon_{r}$ is the permittivity of the medium, $\epsilon_{0}$ is the free space permittivity, $\epsilon_{r}=\epsilon_{\infty}+\frac{\epsilon_{\mathrm{S}}-\epsilon_{\infty}}{1+\jmath 2 \pi f \tau_{\mathrm{D}}}-\jmath \frac{\sigma}{2 \pi f \epsilon_{0}}$ is the complex relative permittivity of medium, $\epsilon_{\infty}$ is the optical relative permittivity, $\epsilon_{\mathrm{S}}$ is the static relative permittivity, $\tau_{\mathrm{D}}$ is the relaxation time, $\sigma$ is the static conductivity.

(8) can be rewritten as

$$
\begin{aligned}
E[n] & =e^{\jmath 2 \pi f \cdot n \Delta t} e^{-j 2 \pi f \sqrt{\epsilon_{r} \frac{d[n]}{C}}} \\
& =e^{\jmath 2 \pi f \cdot n \Delta t} e^{-\jmath 2 \pi f \Re\left[\sqrt{\left.\epsilon_{r}\right] \frac{d[n]}{C}}\right.} e^{2 \pi f \Im\left[\sqrt{\left.\epsilon_{r}\right]} \frac{d[n]}{C}\right.} \\
& \triangleq e^{\jmath 2 \pi f \cdot n \Delta t} \cdot \Theta \cdot \Gamma
\end{aligned}
$$

where $C$ is the speed of light $C=\frac{1}{\sqrt{\epsilon_{0} \mu_{0}}}$ in the vacuum, $\Re\left[\sqrt{\epsilon_{r}}\right]$ and $\Im\left[\sqrt{\epsilon_{r}}\right]$ are the real and the imaginary parts of $\sqrt{\epsilon_{r}}, \Gamma$ is the attenuation defined as

$$
\Gamma[n, f]=e^{2 \pi f \Im\left[\sqrt{\left.\epsilon_{r}\right]} \frac{d[n]}{C}\right.}
$$

and $\Theta$ is the phase shift defined as

$$
\Theta[n, f]=e^{-\jmath 2 \pi f \Re\left[\sqrt{\left.\epsilon_{r}\right] \frac{d[n]}{C}} .\right.}
$$

Our filter is obtained from (10) and (11) as

$$
\begin{aligned}
H\left[n, a_{j}\right] & =\frac{1}{\Theta\left[n, a_{j}\right] \cdot \Gamma\left[n, a_{j}\right]} \\
& =e^{j 2 \pi \frac{f_{c}}{a_{j}} \Re\left[\sqrt{\epsilon_{r}} \frac{d[n]}{C}\right.} e^{-2 \pi \frac{f_{c}}{a_{j}} \Im\left[\sqrt{\left.\epsilon_{r}\right] \frac{d[n]}{C}}\right.}
\end{aligned}
$$

where $a_{j}$ is a scaling factor which controls the actual frequency $f\left(\triangleq \frac{f_{c}}{a_{j}}\right)$ of the filter in order to have a variable window size [19] and $f_{c}$ is the central frequency of $x[n] . H\left[n, a_{j}\right]$ uses $\epsilon_{r}$ of the dominant tissue in the propagating media at $f_{c}$. $H\left[n, a_{j}\right]$ is an exponential function which amplifies the noise in compensated signal and thus causes instability. Therefore $H$ should be stabilized [27] as

$$
H_{s}\left[n, a_{j}\right]=\frac{\Gamma\left[n, a_{j}\right]}{\Gamma\left[n, a_{j}\right]^{2}+T} \cdot \frac{1}{\Theta\left[n, a_{j}\right]}
$$

where $H_{s}$ is the stabilized filter and $T$ is the stabilization factor. The system is stable with a fixed value of $T=10^{-3}$ for all our simulations. Alternatively we can adaptively set $T$ as [19]

$$
T=\frac{\sigma_{g}^{2}}{\sigma_{s}^{2}}
$$

where $\sigma_{g}$ and $\sigma_{s}$ are the variances for the noise and the signal respectively. By applying $H_{s}\left[n, a_{j}\right]$ to $X\left[n, a_{j}\right]$, we obtain the

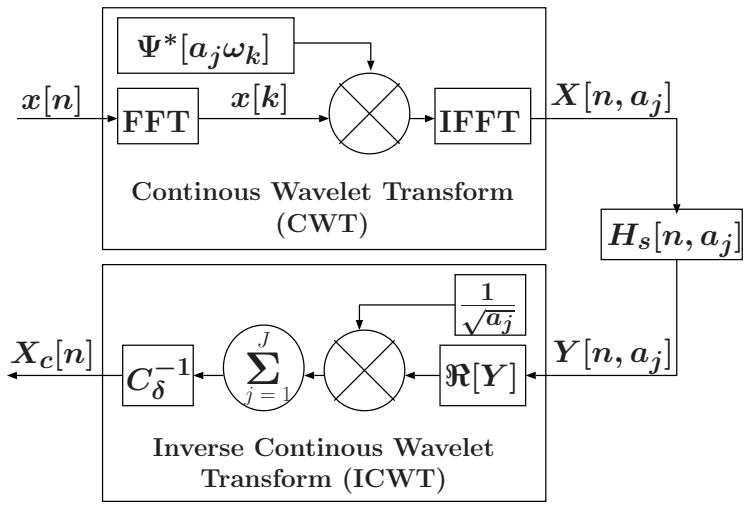

Fig. 1. Compensation method for the dispersive attenuation in the wavelet domain where $\otimes$ means multiplication in frequency domain and corresponds to convolution in time domain.

stabilized compensated wave $Y\left[n, a_{j}\right]$ in the wavelet domain as

$$
Y\left[n, a_{j}\right]=X\left[n, a_{j}\right] H_{s}\left[n, a_{j}\right] .
$$

To avoid the amplification of the high frequency noise, $H_{s}\left[n, a_{j}\right]$ is set to 1 for $0 \leq j \leq \frac{J}{2}$ (short window) which affects the higher part of the spectrum. Finally the compensated signal $X_{c}[n]$ is obtained by applying the inverse continuous wavelet transform (ICWT) to $Y\left[n, a_{j}\right]$ as [23]

$$
X_{c}[n]=\frac{1}{C_{\delta}} \sum_{j=1}^{J} \frac{\Re\left[Y\left[n, a_{j}\right]\right]}{\sqrt{a_{j}}}
$$

where $C_{\delta}$ is a constant for each wavelet function. It is calculated as

$$
C_{\delta}=\sum_{j=1}^{J} \frac{\Re\left[X_{\delta}\left[a_{j}\right]\right]}{\sqrt{a_{j}}}
$$

for the Morlet wavelet where $X_{\delta}$ is the CWT of a delta function $(\delta)$ as

$$
X_{\delta}\left[a_{j}\right]=\frac{1}{N} \sum_{k=1}^{N} \Psi^{*}\left(a_{j} \omega_{k}\right) .
$$

The parameters for the CWT and the ICWT were selected based on the theory in [23]. Our proposed method is depicted in Fig. 1.

\section{CANONicAl TEST}

We expect that the proposed method improves the localization and the resolution of the conventional TR imaging because the inverse filters should compensate the losses caused by the dispersive media. We evaluate the accuracy and the resolution of our method in a random medium [28] to increase the complexity of the propagating medium. The random medium is of Gaussian distribution and the spatial random relative permittivity is defined as

$$
\epsilon_{r n d}[x, y, f]=\epsilon_{r}[x, y, f]+G[x, y]
$$

where $\epsilon_{r}$ is calculated using the one-pole Debye [29] parameters of the muscle tissue [30][31], [x,y] is the Cartesian coordinates in the finite difference time domain (FDTD) space, 


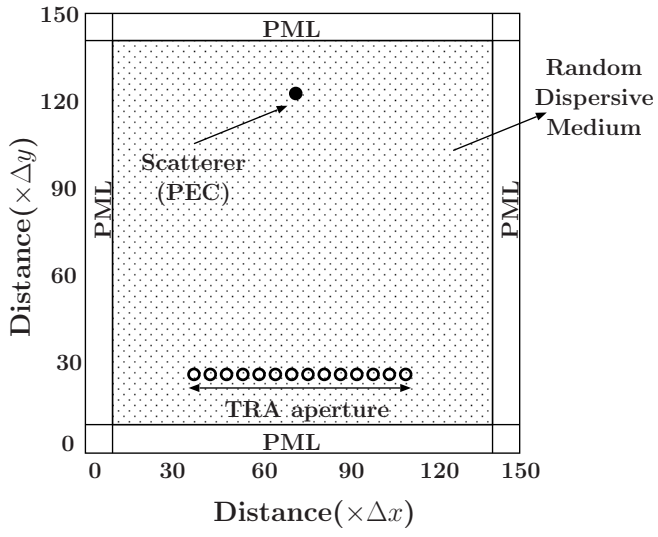

Fig. 2. The simulations setups.

$G$ is a function of space defining the random fluctuation on $\epsilon_{r n d}[x, y]$ and is calculated as

$$
\begin{array}{r}
G[x, y]=\frac{1}{R_{x} R_{y}} \sum_{u=1}^{R_{x}} \sum_{v=1}^{R_{y}}\left(\sqrt{\sum_{x=1}^{R_{x}} \sum_{y=1}^{R_{y}} L[x, y] e^{-\jmath 2 \pi\left(\frac{u x}{R_{x}}+\frac{v y}{R_{y}}\right)}}\right. \\
\left.\cdot \sum_{x=1}^{R_{x}} \sum_{y=1}^{R_{y}} \mathcal{R} \cdot e^{-\jmath 2 \pi\left(\frac{u x}{R_{x}}+\frac{v y}{R_{y}}\right)}\right) e^{\jmath 2 \pi\left(\frac{u x}{R_{x}}+\frac{v y}{R_{y}}\right)}
\end{array}
$$

where $R_{x} \times R_{y}$ is the 2D FDTD size, $[u, v]$ is the Cartesian coordinates in the spatial frequency domain, $\mathcal{R}$ is a Gaussian random number with zero mean and probability density function of $\frac{1}{\sqrt{2 \pi \eta}} e^{\frac{-\zeta^{2}}{2 \eta}}, \zeta$ is a random variable, $\eta$ is the variance and $L$ is the correlation function between $\epsilon_{r}$ at two different spatial points $\left[x_{1}, y_{1}\right]$ and $\left[x_{2}, y_{2}\right]$ given by a Gaussian function as

$$
L[x, y]=\eta e^{\left(-\frac{\left|x_{1}-x_{2}\right|^{2}+\left|y_{1}-y_{2}\right|^{2}}{l_{s}^{2}}\right)}
$$

where $l_{s}$ is the transverse correlation length. Gaussian function is chosen for its generalization and mathematical properties [28]. The random media is characterized by $l_{s}$ and $\eta$. We chose muscle tissue because it is one of the most dispersive tissues in the human body.

\section{A. Radio Environment Settings}

The simulation setup is depicted in Fig. 2. The simulation uses the $\mathrm{TM}_{z}$ polarized 2D FDTD method. The FDTD space is $150 \times 150$ uniformly sampled with the spatial step of $\Delta s \triangleq \Delta x=\Delta y=1 \mathrm{~mm}$. The time step $\Delta t$ is set to 2.36ps. The CFS-PML absorbing boundary conditions [32] are used with 9-cell layer to mimic open space settings. A TRA composed of 15 transceivers antennas are equally spaced from each other and located parallel to the $x$-axis. The TRA has the aperture of $70 \Delta s$. The first TRA antenna is located at $(40 \Delta x, 25 \Delta y)$ and the 15 th is located at $(110 \Delta x, 25 \Delta y)$. A round perfect electric conductor (PEC) scatterer with a $2 \mathrm{~mm}$ radius is centered at $(75 \Delta x, 120 \Delta y)$. The 8th antenna of the TRA transmits a Gaussian pulse modulated at $3 \mathrm{GHz}$ covering from $1.72 \mathrm{GHz}$ to $4.2 \mathrm{GHz}$.

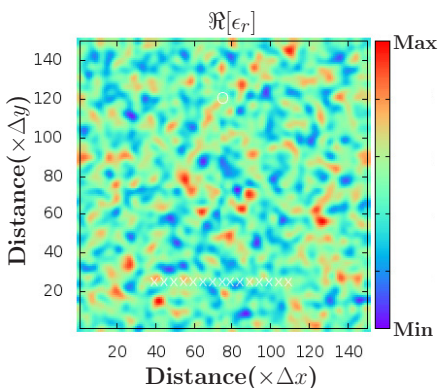

(a) $\left(l_{s}, \eta\right)=\left(4 \Delta s, 0.14 \epsilon_{\infty}\right)$ with minimum and maximum

values of 15.04 and 42.16 respectively.

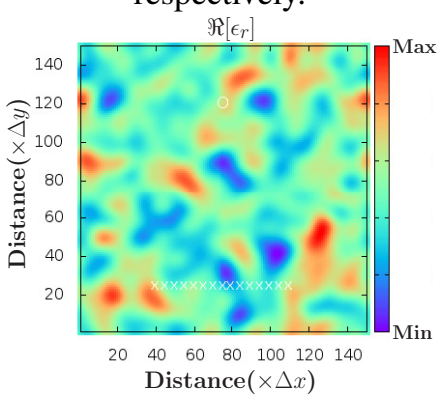

(c) $\left(l_{s}, \eta\right)=\left(10 \Delta s, 0.14 \epsilon_{\infty}\right)$ with minimum and maximum values of 16.88 and 39.51 respectively.

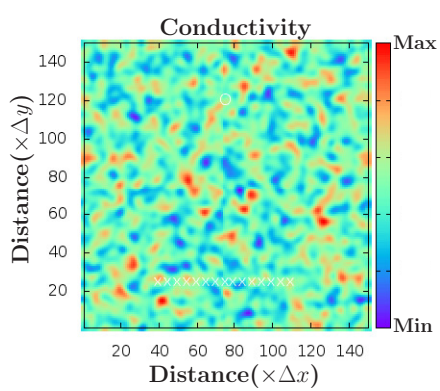

(b) $\left(l_{s}, \eta\right)=\left(4 \Delta s, 0.14 \epsilon_{\infty}\right)$ with minimum and maximum values of $0.38[\mathrm{~S} / \mathrm{m}]$ and 1.13

$[\mathrm{S} / \mathrm{m}]$ respectively.

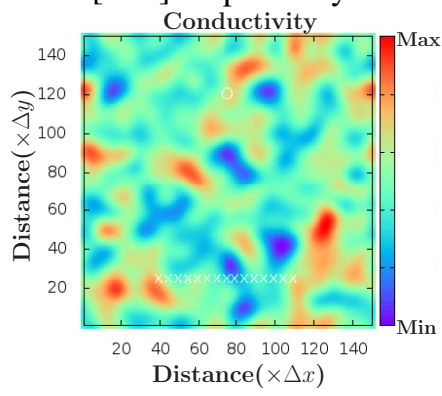

(d) $\left(l_{s}, \eta\right)=\left(10 \Delta s, 0.14 \epsilon_{\infty}\right)$ with minimum and maximum values of $0.44[\mathrm{~S} / \mathrm{m}]$ and 1.06 $[\mathrm{S} / \mathrm{m}]$ respectively.

Fig. 3. The distribution of $\epsilon_{r}$ for Fig. 2 at $3 \mathrm{GHz}$.

\begin{tabular}{|c|c|c|}
\hline$\left(l_{s}, \eta\right)$ & $\frac{R_{t}}{R_{c}}$ & $\frac{R_{s}}{R_{c}}$ \\
\hline$\left(4 \Delta s, 0.14 \epsilon_{\infty}\right)$ & 3.13 & 3.76 \\
\hline$\left(10 \Delta s, 0.14 \epsilon_{\infty}\right)$ & 1.51 & 3.34 \\
\hline \multicolumn{2}{|c|}{ TABLE I }
\end{tabular}

THE SPATIAL RESOLUTION RATIO.

\section{B. Numerical Results}

Fig. 3 shows the example of the spatial distribution of $\epsilon_{r}$ for Fig. 2 at $3 \mathrm{GHz}$ when $l_{s}$ is set to $4 \Delta s$ and $10 \Delta s$. Fig. 4 (a), (b) and (c) show $\left|E_{z}\right|$ in the $x y$-plane with conventional TR, with the method in [12] and with our compensation method. Fig. 5 (a) and (b) show the cross-section of Fig. 4 at $y=120 \Delta y$ with $\left(l_{s}, \eta\right)=\left(4 \Delta s, 0.14 \epsilon_{\infty}\right)$ and the one with $\left(l_{s}, \eta\right)=\left(10 \Delta s, 0.14 \epsilon_{\infty}\right)$ respectively. $R_{c}=\left(x_{4}-x_{3}\right) \Delta x$, $R_{s}=\left(x_{5}-x_{2}\right) \Delta x$ and $R_{t}=\left(x_{6}-x_{1}\right) \Delta x$ represent the resolution of the spatial focusing with the proposed compensation method, the method in [12] and without any compensation method respectively where $x_{i}(1 \leq i \leq 6)$ are on the cross-range and $\left|E_{z}\left(x_{i}\right)\right|=0.5$ for $1 \leq i \leq 6$ where $E_{z}$ is normalized and 0.5 is the cross-range at half maximum to calculate the resolution of the spatial focusing. The spatial resolution of the proposed method is $\left(\frac{R_{s}}{R_{c}}=\right) 3.76$ (Fig. 5(a)),3.34 (Fig. 5(b)) times higher than the compensation method in [12] and $\left(\frac{R_{t}}{R_{c}}=\right) 3.13$ (Fig. 5(a)), 1.51 (Fig. 5(b)) times higher than without using any compensation method as summarized in Table I. Fig. 6 shows the cross-section 


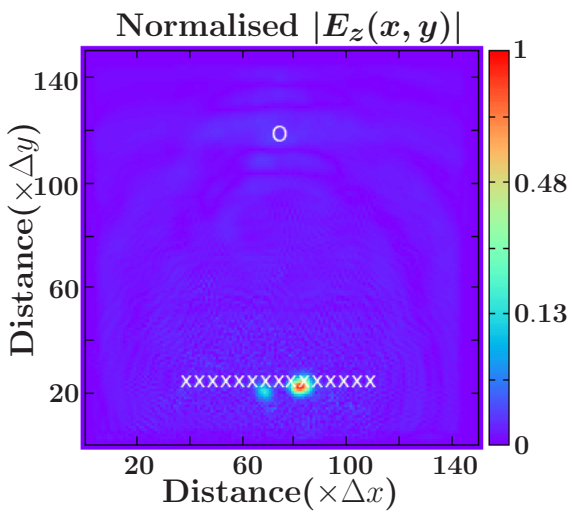

(a) Conventional TR.

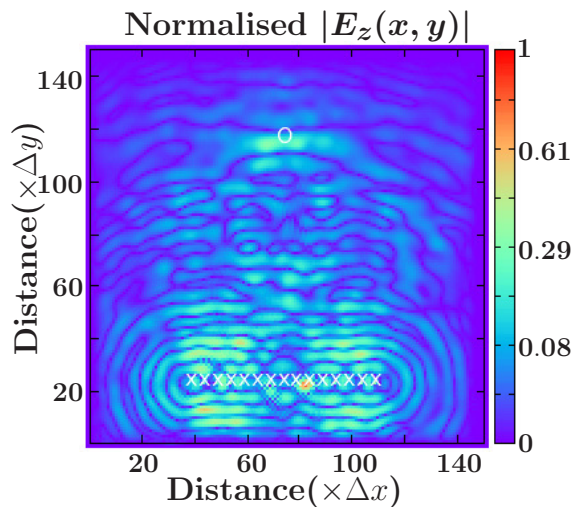

(b) TR with STFT.

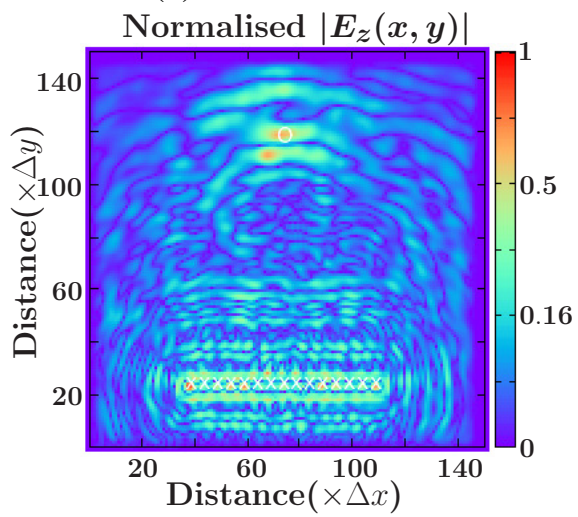

(c) TR with CWT.

Fig. 4. The spatial distribution of $\left|E_{z}\right|$ in the $x y$-plane for the canonical test with conventional TR (without applying any compensation method), with the method in [12] (STFT) and with our compensation method (CWT) when $\left(l_{s}, \eta\right)=\left(4 \Delta s, 0.14 \epsilon_{\infty}\right)$. The scatterer and the TRA antennas are represented by "o" and "x" respectively. $\Delta x=\Delta y=1 \mathrm{~mm}$.

at $y=120 \Delta y$ with our compensation method for the cases $\left(l_{s}, \eta\right)=\left(4 \Delta s, 0.14 \epsilon_{\infty}\right),\left(10 \Delta s, 0.14 \epsilon_{\infty}\right)$ and $\left(4 \Delta s, 0.05 \epsilon_{\infty}\right)$. TR techniques utilize the multipaths components generated by the random media to achieve more accurate focusing resolution than in homogeneous media. Both the high correlation length $l_{s}$ such as $\left(10 \Delta s, 0.14 \epsilon_{\infty}\right)$ and the low variance $\eta$ such as $\left(4 \Delta s, 0.05 \epsilon_{\infty}\right)$ reduce the randomness of the medium as both cases result in reduction of the overall fluctuations of the permittivity of the propagation medium, hence its scattererness. Reducing the randomness decreases the multipath components which lowers the resolution of the TR focusing.

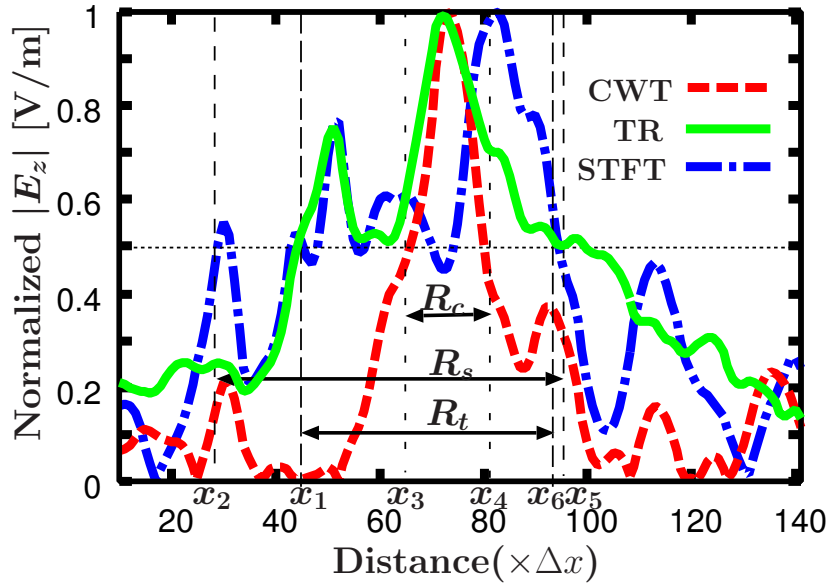

(a) $\left(l_{s}, \eta\right)=\left(4 \Delta s, 0.14 \epsilon_{\infty}\right)$.

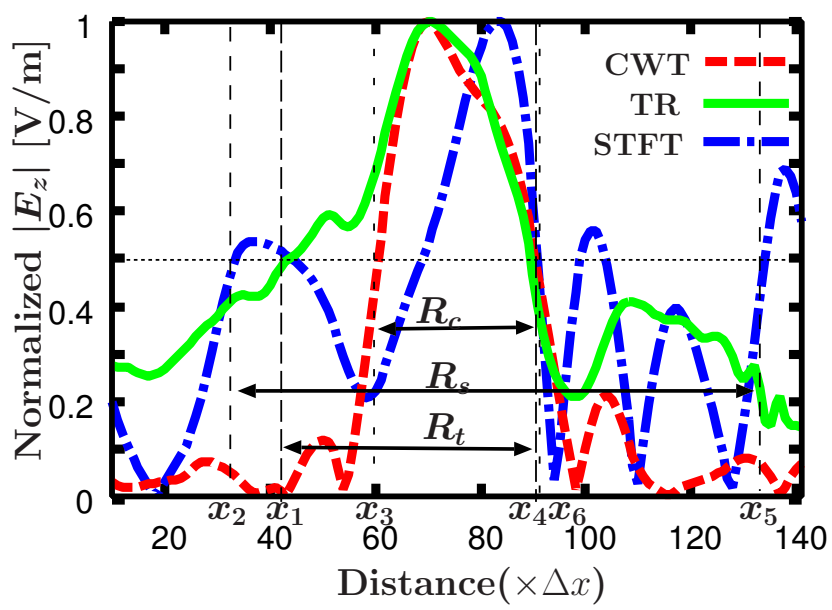

(b) $\left(l_{s}, \eta\right)=\left(10 \Delta s, 0.14 \epsilon_{\infty}\right)$.

Fig. 5. The cross-section of the normalized $\left|E_{z}\right|$ distribution in Fig. 4 at $y=120 \Delta y$ in the FDTD space after applying CWT, STFT and without applying any compensation method.

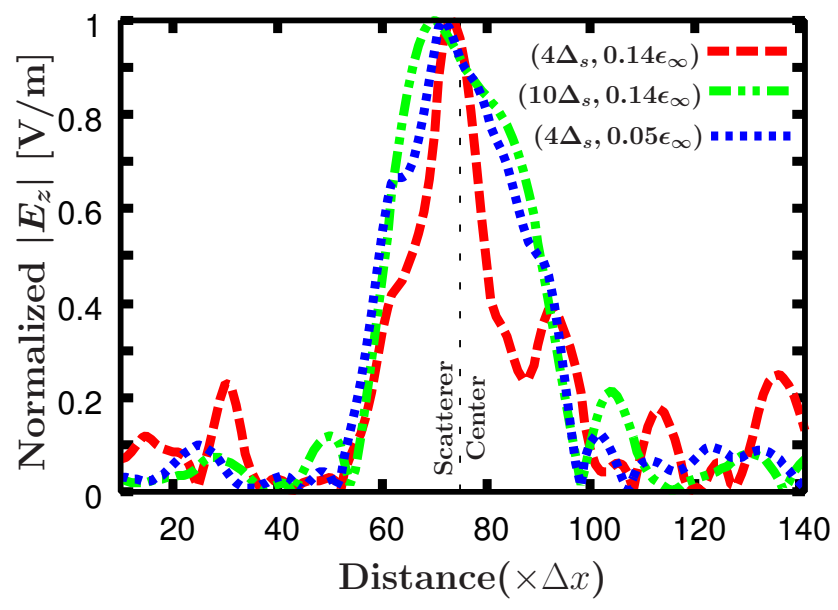

Fig. 6. The cross-section of the normalized $\left|E_{z}\right|$ distribution at $y=$ $120 \Delta y$ in the FDTD space after applying CWT for the cases $\left(l_{s}, \eta\right)=$ $\left(4 \Delta s, 0.14 \epsilon_{\infty}\right),\left(10 \Delta s, 0.14 \epsilon_{\infty}\right)$ and $\left(4 \Delta s, 0.05 \epsilon_{\infty}\right)$. 


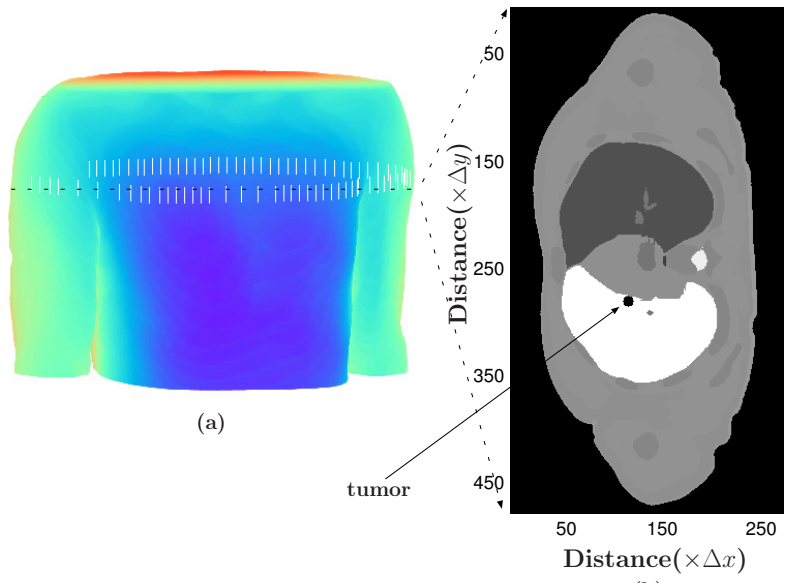

(b)

Fig. 7. The human phantom with $5 \mathrm{~mm}$ radius round tumor. The TRA antennas are represented by "|". $\Delta x=\Delta y=\Delta z=1 \mathrm{~mm}$.

When the computational times between our proposed method and original STFT method of [12] are compared, it is not merely comparison between the STFT and CWT operations but the whole algorithm implementation starting from the filter generations. The way [12] used to produce the inverse filter took the major part of the simulation time because the creation of the inverse filters in [12] needs two FDTD calculations while our approach with CWT does not require any FDTD calculations. Applying different FDTD sizes with different simulation times shows that the proposed method is 1.8 times faster than the STFT method in [12] and [17] in average.

\section{PRACTICAL APPLICATION}

Lung cancer is identified as one of the deadliest cancers affecting people [33], [34]. Early-stage lung cancer is difficult to detect because the tumor is small and its electromagnetic properties are close to the parameters of the healthy lung. We apply the TR technique with our proposed compensation method to locate an early-stage tumor in a digital human phantom (DHP). The DHP was provided by RIKEN (Saitama,Japan) under non-disclosure agreement between RIKEN and the University of Manchester. The usage was approved by RIKEN ethical committee. The DHP has $1 \mathrm{~mm}$ resolution and contains 52 segmented tissue. We fitted the one-pole Debye parameters of human tissues [35] using the measurement provided by [36], [37]. The Debye media parameters for human tissues are presented in [31]. The purpose of this numerical simulation is to evaluate the accuracy and the resolution of our method in the human phantom medium.

\section{A. Radio Environment Setting}

Fig. 7 shows the DHP with an early stage tumor represented by a round scatterer with a $5 \mathrm{~mm}$ radius centered at $(115 \Delta x, 285 \Delta y, 95 \Delta z)$. The FDTD space is $265 \times 490 \times 290$ including the CFS-PML cells. The 102 TRA $z$-directed 14 $\mathrm{mm}$ dipole antennas [38] are placed in the fat tissue assuming that we use the implantable antennas [39], [40], [41]. The human tissues are represented using the one-pole Debye model.

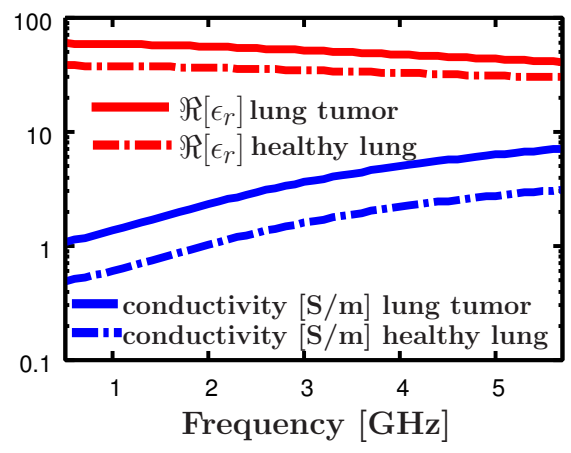

Fig. 8. Relative permittivity and the conductivity of the lung and the scatterer

We shifted the frequency response of the healthy lung tissue to match the complex permittivity of the lung cancer tissue presented in [42] and generated the Debye parameters for the lung tumor by data-fitting of the modified frequency response. Fig. 8 shows the relative permittivity and the conductivity of the lung and the tumor. The size and the Debye parameters of the tumor are set to mimic an early stage cancer. The antenna at $(31 \Delta x, 281 \Delta y, 95 \Delta z)$ transmits a Gaussian pulse modulated at $3 \mathrm{GHz}$. The remaining simulation settings are the same as in Section III-A.

\section{B. Numerical Results}

Fig. 9 shows $\left|E_{z}\right|$ on $z=95 \Delta z$ plane within the 3D DHP with conventional TR without applying any compensation, one with the method given in [12] (STFT) and one with our method (CWT). Fig. 9 (b) shows that the method in [12] did not improve the conventional TR imaging or locate the tumor correctly. The inverse filters utilized in [12] not only amplify the signal of interest, but also the noise. This reduces the signal to noise ratio (SNR) and in return lowers the accuracy of the localization. The proposed CWT filters compensate the losses caused by the dispersive tissues. Therefore application of the proposed CWT method to the TR technique gives more accurate spatial focusing than both the conventional TR and the method in [12].

\section{CONCLUSION}

The wave equation invariance of the time reversal technique is broken in human tissues due to their dispersive characteristics. Since the dispersive attenuation is both duration and frequency dependent, we have introduced CWT based compensation method that uses inverse filters in the wavelet domain to overcome this attenuation. The inherent time and frequency variations in a wavelet decompose the recorded signals into different time and frequency components to which different filters would be applied for compensation. CWT does not need optimization of the time-window length as is the case with STFT. The proposed inverse filters need only the complex permittivity at the center frequency of one dominant medium to create the inverse filter as opposed to the whole wide-band dispersion characteristic in the STFT method [12]. Therefore CWT technique can be applied in real-life scenarios while STFT method [12] [17] needs the non-dispersive version of the 


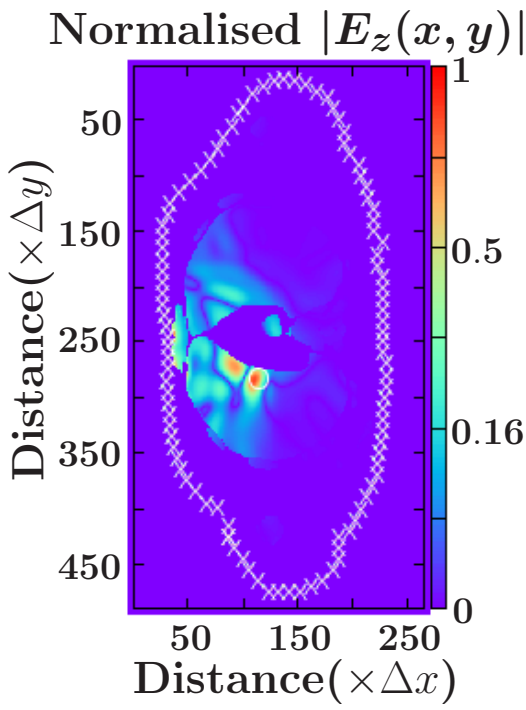

(a) TR with CWT.

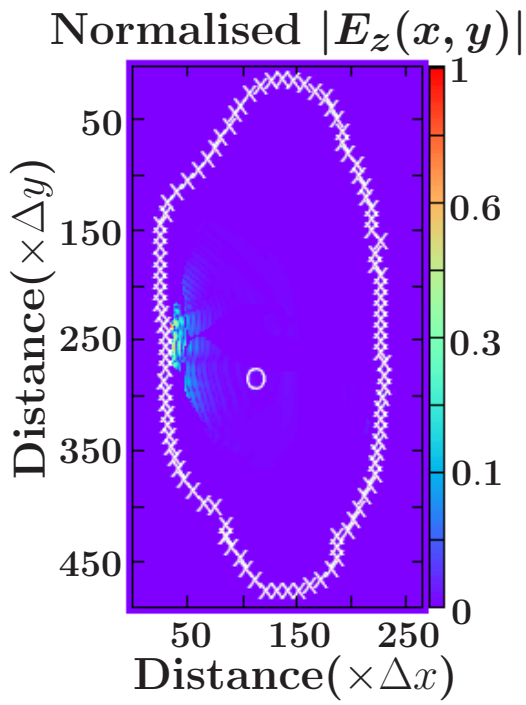

(b) TR with STFT.

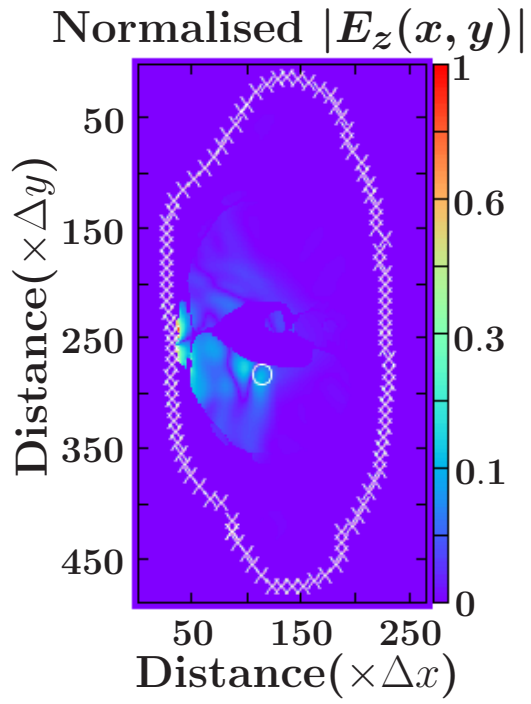

(c) TR.

Fig. 9. The spatial distribution of $\left|E_{z}\right|$ on $z=95 \Delta z$ plane for the human phantom with our compensation method, the method in [12] and without any compensation method. The scatterer and the TRA antennas are represented by "o" and "x" respectively. $\Delta x=\Delta y=\Delta z=1 \mathrm{~mm}$. In order to present $\left|E_{z}\right|$ inside the lung clearly, $\left|E_{z}\right|$ on the skin, muscle and fat are removed from the figures. propagation media to create the inverse filter (in real-life, such an information might not be available readily). We applied our compensation method to the TR signals in different scenarios to examine how our method improved the TR imaging. In the canonical numerical simulation, our compensation method improved the spatial focusing of the TR technique. The TR focusing around the object location is more accurate with our proposed method than those with the method in [12] or without applying any method (i.e. with conventional TR imaging). Furthermore, the resolution is 3.76 and 3.13 times higher with the proposed method than with the method in [12] or without any compensation method respectively. The tumor is located accurately after applying our proposed compensation method. An additional advantage of the CWT-based method is that it is 1.8 times faster than the STFT method in average.

\section{ACKNOWLEDGMENT}

"Wavelet software was provided by C. Torrence and G. Compo, and is available at URL: http://paos.colorado.edu/research/wavelets/'. The base code to produce Fig. 7 (a) was provided by one of our research group members, Mr. K. Tekbas.

\section{REFERENCES}

[1] P. D. Gader, M. Mystkowski, and Y. Zhao, "Landmine detection with ground penetrating radar using hidden markov models," IEEE Trans. Geosci. Remote Sens., vol. 39, No. 6, pp. 1231-1244, June 2001.

[2] P. Pratihar and A. K. Yadav, "Detection techniques for human safety from concealed weapon and harmful EDS," International Review of Applied Eng. Research., vol. 4, No. 1, pp. 71-76, 2014.

[3] M. E. Yavuz, A. E. Fouda, and F. L. Teixeira, "GPR signal enhancement using sliding-window space-frequency matrices," Progress In Electromagnetics Research, vol. 145, pp. 1-10, 2014.

[4] W. Shao, B. Zhou, Z. Zheng, and G. Wang, "UWB microwave imaging for breast tumor detection in inhomogeneous tissue," Eng. in Med. and Biol. 27th Annual Conference, pp. 1496-1499, 2005.

[5] M. Hossain, A. Mohan, and M. Abedin, "Beamspace time-reversal microwave imaging for breast cancer detection," IEEE Antennas Wireless Propag. Lett., vol. 12, pp. 241-244, 2013.

[6] N. Ray, B. Saha, and M. Graham Brown, "Locating brain tumors from MR imagery using symmetry," in 2007 Conference Record of the FortyFirst Asilomar Conference on Signals, Systems and Computers (ACSSC), pp. 224-228, Nov 2007.

[7] A. Zamani, S. Rezaeieh, and A. Abbosh, "Lung cancer detection using frequency-domain microwave imaging," Electronics Lett., vol. 51, no. 10 , pp. $740-741,2015$.

[8] M. Fink, D. Cassereau, A. Derode, C. Prada, P. Roux, M. Tanter, J. Thomas, and F. Wu, "Time-reversed acoustics," Rep. Prog. Phys., vol. 63, pp. 1933-1995, 2000.

[9] C.-X. Li, W. Xu, J.-L. Li, and X.-Y. Gong, "Time-Reversal detection of multidimensional signals in underwater acoustics," IEEE J. Ocean. Eng., vol. 36, pp. 60-70, Jan 2011.

[10] R. Solimene, G. Leone, and A. Dell'Aversano, "MUSIC algorithms for rebar detection," J. Geophysics and Eng., vol. 10, No. 6, 2013.

[11] A. E. Fouda, F. L. Teixeira, and M. E. Yavuz, "Time-reversal techniques for MISO and MIMO wireless communication systems," Radio Science, vol. 47, 2012.

[12] M. E. Yavuz and F. L. Teixeira, "Frequency dispersion compensation in time reversal techniques for UWB electromagnetic waves," IEEE Geosci. Remote Sens. Lett., vol. 2, no. 2, pp. 233-237, April 2005.

[13] M. E. Yavuz and F. L. Teixeira, "Full time-domain DORT for ultrawideband electromagnetic fields in dispersive, random inhomogeneous media," IEEE Trans. Antennas Propag., vol. 54, pp. 2305-2315, Aug 2006.

[14] M. Tanter, J. L. Thomas, and M. Fink, "Focusing and steering through absorbing and aberrating layers: Application to ultrasonic propagation through the skull," J. Acoust. Soc. Am., vol. 103, pp. 2403-2410, May 1998. 
[15] T. Folegot, C. Prada, and M. Fink, "Resolution enhancement and separation of reverberation from target echo with the time reversal operator decomposition," J. Acoust. Soc. Am., vol. 113, No. 6, pp. 31553160, 2003.

[16] M. Saillard, P. Vincent, and G. Micolau, "Reconstruction of buried objects surrounded by small inhomogeneities," Inverse Problems, vol. 16, pp. 1195-1208, 2000.

[17] A. M. Abduljabbar, M. E. Yavuz, F. Costen, R. Himeno, and H. Yokota, "Frequency dispersion compensation through variable window utilization in time reversal techniques for electromagnetic waves," IEEE Trans. Antennas Propagat., vol. 64, pp. 3636-3639, Aug 2016.

[18] C. Reine, M. Van Der Baan, and R. Clark, "The robustness of seismic attenuation measurements using fixed- and variable-window timefrequency transforms," Geophysics, vol. 74, No. 2, pp. WA123-WA135, 2009.

[19] I. Braga and F. Moraes, "High-resolution gathers by inverse Q filtering in the wavelet domain," Geophysics, vol. 78, No. 2, pp. V53-V61, 2013.

[20] I. Hostens, J. Seghers, A. Spaepen, and H. Ramon, "Validation of the wavelet spectral estimation technique in biceps brachii and brachioradialis fatigue assessment during prolonged low-level static and dynamic contractions," J. Electromyography and Kinesiology, vol. 14, no. 2, pp. $205-215,2004$.

[21] M. E. Yavuz and F. L. Teixeira, "Ultrawideband microwave sensing and imaging using time-reversal techniques: A review," Remote Sens., vol. 1, no. 3, pp. 466-495, 2009.

[22] J. Morlet, G. Arens, E. Fourgeau, and D. Giard, "Wave propagation and sampling theory-Part I: Complex signal and scattering in multilayered media," Geophysics, vol. 47, No. 2, pp. 203-221, 1982.

[23] C. Torrence and G. P. Compo, "A practical guide to wavelet analysis," Bulletin of the American Meteorological Soc., vol. 79, No.1, pp. 61-78, 1998.

[24] M. Farge, "Wavelet transforms and their applications to turbulence," Annu. Rev. Fluid Mech, vol. 24, pp. 395-457, 1992.

[25] H. Q. Ngo, E. G. Larsson, and T. L. Marzetta, "The multicell multiuser MIMO uplink with very large antenna arrays and a finite-dimensional channel," IEEE Trans. Commun., vol. 61, pp. 2350-2361, June 2013.

[26] A. Taflove and S. Hagness, Computational Electromagnetics. The finitedifference Time-domain method. Artech House, 2005.

[27] Y. Wang, "Inverse Q-filter for seismic resolution enhancement," Geophysics, vol. 71, pp. V51-V60, 2006.

[28] C. Moss, F. Teixeira, Y. Yang, and J. A. Kong, "Finite-difference timedomain simulation of scattering from objects in continuous random media," IEEE Trans. Geosci. Remote Sens., vol. 40, pp. 178-186, Jan 2002.

[29] P. Debye, Polar Molecules. New York: Dover, 1929.

[30] M. Abalenkovs, F. Costen, J.-P. Bérenger, R. Himeno, H. Yokota, and M. Fujii, "Huygens subgridding for 3-D frequency-dependent finitedifference time-domain method," IEEE Trans. Antennas Propag., vol. 60, No. 9, pp. 4336-4344, September 2012.

[31] RIKEN, Wako Saitama, Japan, "Media parameters for the Debye relaxation model." http://cfd-duo.riken.jp/cbms-mp/. [Online; accessed 26April-2016].

[32] J.-P. Bérenger, "Numerical reflection from FDTD-PMLs: A comparison of the split PML with the unsplit and CFS PMLs," IEEE Trans. Antennas Propag., vol. 50, No. 3, pp. 258-265, 2002.

[33] W. Weber, Pharmacogenetics. Oxford University Press, Apr 2008.

[34] N. Emaminejad, W. Qian, Y. Guan, M. Tan, Y. Qiu, H. Liu, and B. Zheng, "Fusion of quantitative image and genomic biomarkers to improve prognosis assessment of early stage lung cancer patients," IEEE Trans. Biomed. Eng., vol. 63, pp. 1034-1043, May 2016.

[35] T. Wuren, T. Takai, M. Fujii, and I. Sakagami, "Effective 2-debye-pole fdtd model of electromagnetic interaction between whole human body and uwb radiation," IEEE Microw. Wireless Compon. Lett., vol. 17, pp. 483-485, July 2007.

[36] C. Gabriel, S. Gabriel, and E. Corthout, "The dielectric properties of biological tissues: I. Literature survey," Physics in Med. and Biol., vol. 41, pp. 2231-2249, 1996.

[37] R. W. L. S. Gabriel and C. Gabriel, "The dielectric properties of biological tissues: II. Measurements in the frequency range $10 \mathrm{~Hz}$ to 20 GHz," Physics in Med. and Biol., vol. 41, pp. 2251-2269, 1996.

[38] Y. Chen and P. Kosmas, "Detection and localization of tissue malignancy using contrast-enhanced microwave imaging: Exploring information theoretic criteria," IEEE Trans. Biomed. Eng., vol. 59, pp. 766-776, March 2012

[39] C. M. Furse and A. Chrysler, "A history \& future of implantable antennas," in 2014 IEEE Antennas and Propag. Soc. International Symposium (APSURSI), pp. 527-528, July 2014.
[40] J. Kim and Y. Rahmat-Samii, "Implanted antennas inside a human body: simulations, designs, and characterizations," IEEE Trans. Microw. Theory Tech., vol. 52, pp. 1934-1943, Aug 2004.

[41] S. Alamri, A. AlAmoudi, and R. Langley, "Gain enhancement of implanted antenna using lens and parasitic ring," Electron. Lett., vol. 52, no. 10, pp. 800-801, 2016.

[42] A. Zamani, S. Rezaeieh, and A. Abbosh, "Lung cancer detection using frequency-domain microwave imaging," Electron. Lett., vol. 51, no. 10, pp. 740-741, 2015.

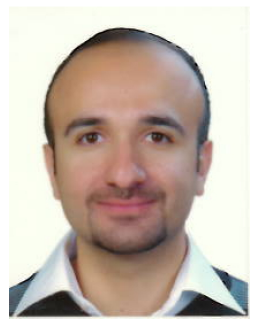

Ammar M. Abduljabbar was born in Baghdad, Iraq, in 1985. He received the B.Sc. degree in computer engineering from the University of Baghdad, Baghdad, Iraq, in 2007, and the M.Sc. degree in wireless communication systems from Brunel University, London, UK, in 2009. In 2013, he started his $\mathrm{PhD}$ study in Electrical and Electronics Engineering, University of Manchester, UK. His current research interests include microwave imaging techniques and high performance computing.

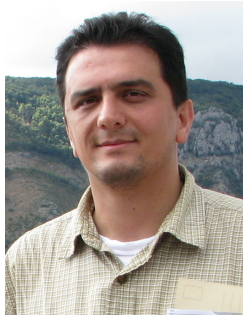

Mehmet E. Yavuz received the B.S. degree from the Middle East Technical University (METU), Ankara, Turkey in 2001, M.S. degree from the Ko University, Istanbul, Turkey in 2003 and the Ph.D. degree from the Ohio State University (OSU) in 2008, all in electrical engineering. He has worked as a Principle Scientist at the Halliburton Energy Services in Houston, TX, as a Visiting Assistant Professor at the Electrical Engineering Department of the American University of Sharjah in the U.A.E and as a Research Scientist at Intel Corporation in Hillsboro, Oregon. His current research interests include computational electromagnetics for industrial applications, radar-based signal processing and microwave imaging and detection.

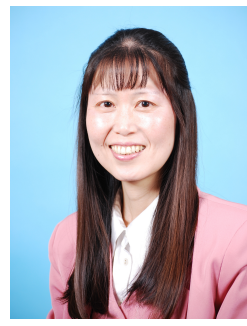

Fumie Costen (M'07) received the B.Sc. degree, the M.Sc. degree in electrical engineering and the Ph.D. degree in Informatics, all from Kyoto University, Japan. From 1993 to 1997 she was with Advanced Telecommunication Research International, Kyoto, where she was engaged in research on direction-of-arrival estimation based on Multiple SIgnal Classification algorithm for 3-D laser microvision. She filed three patents from the research in 1999 in Japan. She was invited to give 5 talks in Sweden and Japan during 1996-2014. From 1998 to 2000, she was with Manchester Computing in the University of Manchester, U.K., where she was engaged in research on metacomputing and has been a Lecturer since 2000. Her research interests include computational electromagnetics in such topics as a variety of the finite difference time domain methods for microwave frequency range and high spatial resolution and FDTD subgridding and boundary conditions. She filed a patent from the research on the boundary conditions in 2012 in the U.S.A. Her work extends to the hardware acceleration of the computation using general-purpose computing on graphics processing units, Streaming Single Instruction Multiple Data Extension and Advanced Vector eXtentions instructions. Dr. Costen received an ATR Excellence in Research Award in 1996 and a best paper award from 8th International Conference on High Performance Computing and Networking Europe in 2000. 


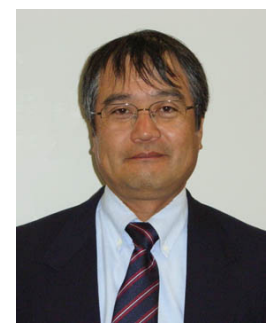

Ryutaro Himeno received his Doctor of Engineering degree from the University of Tokyo in 1988. In 1979, he joined Central Research Laboratories, Nissan Motor Co., Ltd., Yokosuka, Japan, where he has been engaged in the research of applying Computational Fluid Dynamics analysis to the car aerodynamic development. In 1998, he joined RIKEN and is the director of Advanced Center for Computing and Communication and had been the deputy program director of the Next Generation Computational Science Research Program at RIKEN till April, 2013. He is also a visiting professor at Hokkaido University, Kobe University and Tokyo Denki University. He currently studies Computational Bioengineering, High Performance Computing and blood flows of human bodies. He was a winner of Nikkei Science, Computer Visualization Contest in 2000 and Scientific Visualization Contest in 1996, and received JSME Computational Mechanics Division Award in 1997 and JSME Youth Engineer Award in 1988. He has also received the Paper Award by NICOGRAPH in 1993, Giga FLOPS Award by CRAY Research Inc. in 1990 and other awards.

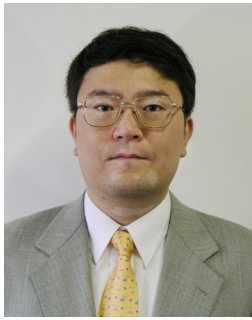

Hideo Yokota received his Doctor of Engineering degree from the University of Tokyo in 1999. In 1993, he joined Higuchi Ultimate Mechatronics Project, Kanagawa Academy of Science and Technology, Kawasaki, Japan. In 1999, he joined RIKEN and is the contract researcher of Computational biomechanics unit. 2004-2012 Bio-research Infrastructure Construction Team Leader, VCAD system research program, RIKEN. 2007-2012 Cell-scale Research and Development Team Leader,Research Program for Computational Science, RIKEN. 2013present Image Processing Research Team Leader, Center for Advanced Photonics,RIKEN. He is also a visiting Professor at Hokkaido University, Kobe University, Tokai University and the Tokyo University of Agriculture and Technology. He currently studies biomedical imaging and image processing to the biomedical simulation. Bioimaging Society, Best Image Award, 2005. The Commendation for Science and Technology by the Minister of Education, Culture, Sports, Science and Technology, Young Scientists Prize in 2008. 